Research Article

\title{
Outer Macular Microvascular Supply in Retinitis Pigmentosa Examined using Optical Coherence Tomography Angiography
}

\author{
Mingsheng Hong $\mathbb{D}^{1},{ }^{1}$ Jiquan Wen, ${ }^{1}$ Jixian Lou, ${ }^{1}$ Jiehui $\mathrm{Xu},{ }^{1}$ and Tingjun $\mathrm{Xu} \mathbb{D}^{2}$ \\ ${ }^{1}$ Department of Ophthalmology, Zhejiang Hospital, Hangzhou 310013, China \\ ${ }^{2}$ Department of Ophthalmology, The First People's Hospital of PingHu, PingHu 314200, China \\ Correspondence should be addressed to Tingjun Xu; xutingjun1983@163.com
}

Received 28 February 2021; Revised 9 November 2021; Accepted 26 November 2021; Published 21 December 2021

Academic Editor: Monica L. Acosta

Copyright (c) 2021 Mingsheng Hong et al. This is an open access article distributed under the Creative Commons Attribution License, which permits unrestricted use, distribution, and reproduction in any medium, provided the original work is properly cited.

\begin{abstract}
Purpose. To determine the vessel density of the superior (SCP) and deep retinal capillary plexuses (DCP) in patients with retinitis pigmentosa (RP) using optical coherence tomography angiography (OCTA). Methods. This was a cross-sectional study. A total of 25 eyes of 25 healthy volunteers and 30 eyes of 17 patients with RP were evaluated in this study. The integrity of the ellipsoid zone in the macular fovea was evaluated as an intact or defect using a spectral-domain OCT. Commercial spectral domain coherence tomography angiography (OCTA) was used to scan the macular region of approximately $3 \times 3 \mathrm{~mm}^{2}$. The vessel density in the SCP and DCP were calculated after appropriate layer segmentation and removal of projection artifacts. The central retinal thickness (CRT) was measured with automated software. The vessel densities in the SCP and DCP were compared between different groups using SPSS. Results. A total of 25 eyes of 25 healthy subjects and 30 eyes of 17 patients with RP were evaluated in the study. There was no significant difference in ages between the two groups $(F=0.065$ and $P=0.937)$. There was a significant difference in SCP and DCP between the patients with RP and healthy individuals $(P<0.001$ and $P<0.001)$. The DCP was significantly reduced in the parafovea region between the macular intact and defect groups $(P<0.05)$, except in the fovea and nasal regions. After linear regression, the DCP/SCP ratio in the whole, fovea, and parafovea regions was closely related to the DCP vessel density $(P<0.05)$, and CRT in the fovea and parafovea was not related to the whole DCP $(P=0.186$ and $P=0.539)$. Conclusion. The vessel density decreased in patients with RP, especially in the DCP of the parafovea region. A greater loss of capillaries in the DCP was found when the macular region was involved. The DCP/SCP ratio may be an important indicator of RP.
\end{abstract}

\section{Introduction}

Retinitis pigmentosa (RP) is a wide group of hereditary retinal diseases with a prevalence of 1 in 1000-5000 individuals [1]. More than 1 million individuals are affected by the disease [2]. Degeneration of the rods and cones is the main pathological change. RP is one of the leading causes of blindness in elderly individuals aged $<60$ years [3]. The onset is always the constriction of the peripheral visual field and the ultimate loss of central vision [2]. Mutation in rhodopsin has been identified in patients with RP and is regarded as the key mechanism in photoreceptor degeneration [4]. Moreover, retinal and choroid hemodynamics have been found to decrease with impaired retinal vascular oxygen saturation
[5]. Retinal vascular stenosis is a typical manifestation in RP. Therefore, insufficient vascular supply is also another factor in the pathology of RP.

Recent advancements in the noninvasive measurement of retinal blood supply offer new insights into the pathogenesis of patients with RP [6-8]. A previous study found a significant reduction in vessel density (VD) in the whole retinal and perifoveal regions $[6,9]$. However, it did not demonstrate a dynamic change in the disease progression. Another study reported that the retinal vascular supply was strongly reduced in the rat model of RP, especially in the outer retinal structure; however, it did not illustrate the vascular condition when the macular region was involved [10]. The macular dysfunction always occurred at a late stage, 
and the visual acuity (VA) was always poor in patients with RP [11]. However, whether the VD decreased with macular involvement was unknown. Optical coherence tomography angiography (OCTA) has been used to measure the retinal VD to reflect the vascular changes [12]. This study aimed to characterize the alternation of retinal VD in patients with RP and to determine whether the reduction in retinal VD was accompanied by macular dysfunction. The present study indicated that the vessel density is decreased in those with a disrupted ellipsoid zone.

\section{Materials and Methods}

This was a cross-sectional study. Healthy volunteers and patients with RP were recruited from Zhejiang Hospital. Patients with systemic diseases (hypertension, diabetes, and Alzheimer's disease) and other ocular diseases (macular hole, epiretinal membrane, retinal vessel occlusion, glaucoma, age-related macular degeneration, and optic diseases) and patients who previously underwent any ocular surgery were excluded in the study. Written informed consent was provided by the participants. All the participants underwent comprehensive ophthalmologic examinations, including medical history, best-corrected VA measurement using a Snellen chart (VA was changed to logMAR for analysis), slitlamp examination, color fundus photography, fundus autofluorescence (FA) imaging using the TOPCON camera (Topcon Corporation, IMAGEnet 200, Tokyo, Japan), SDOCT of the macular region (Heidelberg), and OCTA using Avanti RTVue-XR platform (Optovue, Fremont, CA, USA).

The diagnosis of RP was finally verified using the fullfield electroretinogram by the RETIport system (Roland Consult, Brandenburg, Germany). The test was conducted according to the standards of the International Society for Clinical Electrophysiology of Vision.

2.1. OCTA Image Acquisition and Analysis. All the subjects underwent OCTA on the macular $3 \times 3 \mathrm{~mm}^{2}$ region. The Avanti RTVue-XR system (Optovue) adopted a split-spectrum amplitude-decorrelation angiography algorithm to extract the OCTA information. The transverse resolution was $5 \mu \mathrm{m}$ and $22 \mu \mathrm{m}$ in the tissues, respectively. The system has a repetition frequency of $70 \mathrm{k}$ A-scans per second. Each scan consisted of volumetric scans with orthogonal directions ( $X$-fast and $Y$-fast). Each volume of OCTA consisted of $304 \times 304$ A-scans. Projection artifacts were removed by the projection-resolved algorithm as previously conducted [13]. Vascular plexuses were separated by the automated segmentation function software, and the manual correction was performed when the macular structure was intact to ensure appropriate segmentation. All images were carefully reviewed by professional operators. The superficial retinal capillary plexus (SCP) was defined as the area $3 \mu \mathrm{m}$ below the internal limiting membrane to $15 \mu \mathrm{m}$ below the inner plexiform layer. The deep capillary plexus (DCP) was defined as the area from 15 to $70 \mu \mathrm{m}$ below the IPL [12-14] (Figure 1). The VD was defined as the percentage area of pixels occupied by the vascular flow signal relative to the total scanning area. The VD was measured from the en face of OCT on the fovea and parafovea. Only the qualified images (signal strength index $>50$ ) were selected for the analysis.

2.2. Statistical Analysis. All data were presented as mean\pm deviation (range) when they have a normal distribution, while number (percentage) was used to describe non-normal distribution. The parametric quantitative data were compared between groups using independent $t$-test or analysis of variance (ANOVA), when necessary. The least significant difference (LSD) was used to compare two groups after ANOVA. The nonparametric data were compared using Kruskal-Wallis $\mathrm{H}$ test, while the distributions of sex in different groups were conducted by the chi-square test. All data were analyzed using IBM SPSS Statistics version 25.0 (IBM Corp. Armonk, NY, USA).

\section{Results}

3.1. Study Demographic Data. A total of 25 eyes from 25 healthy volunteers were recruited in the study. The mean age was $51.0 \pm 12.0$ years (28-66 years). Among them, 10 were male and 15 were female. A total of 25 patients with bilateral RP were enrolled in the study. Moreover, five eyes in three patients with macular epiretinal membrane, one eye in one patient with macular hole, and 14 eyes in 10 patients (four patients with bilateral eyes, six patients with unilateral eye) with poor images were excluded. Only 30 eyes from 17 patients with RP were evaluated in the study. The mean age of the patients was $50.4 \pm 11.5$ years $(32-60$ years). Ten patients were male, and seven were female. We classified them into macular intact or defect groups according to the integrity of the ellipsoid zone in the macular fovea region (Table 1 and Figure 1). If the ellipsoid zone is intact, it belongs to the macular intact group; otherwise, it belongs to the macular defect group. Furthermore, 15 eyes from eight patients with RP belong to the macular defect group, the mean age of the patients was $51.3 \pm 12.4$ years (32-60 years), and the VA was 1.5 (1.2-0.5) (approximately to 20/1000 Snellen equivalent). Fifteen eyes from nine patients belong to the macular intact group, the mean age of patients was $49.0 \pm 10.0$ years (37-57 years), the VA was $0.30(0.30-0)$ (approximately 20/40 Snellen equivalent) in the macular intact group. There was no significant difference in age within the groups ( $F=0.065$ and $P=0.937$, ANOVA) (Table 1$)$. The sex distribution was not significantly different in all groups $(P=0.672)$. VA was better in the macular intact group than in the macular defect group $(P=0.014)$.

3.2. VD and Central Macular Retinal Thickness (CMT) Measured by OCTA. The measurement of VD through analysis of en face OCTA in patients with RP indicated the loss of vascular signal compared to normal individuals (Table 2 and Figure 1). The VD was calculated in the superficial and deep vascular plexuses by automated software. The macular defect group showed greater vessel loss than the 

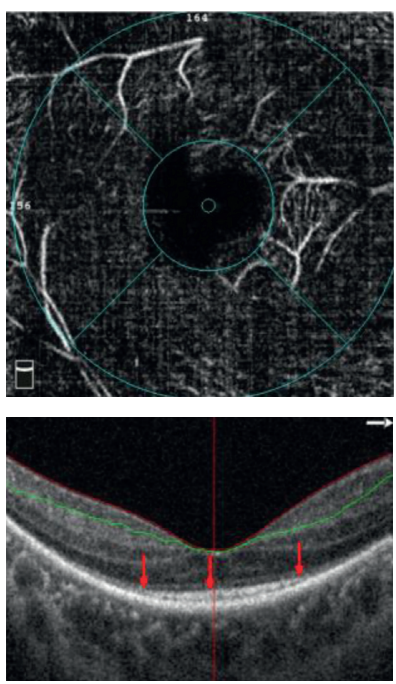

(a)
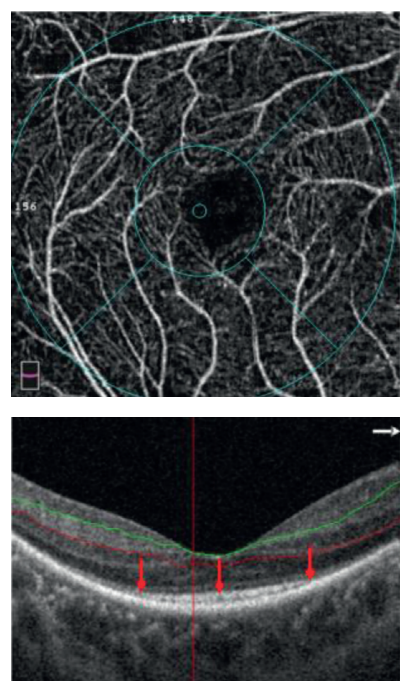

(b)
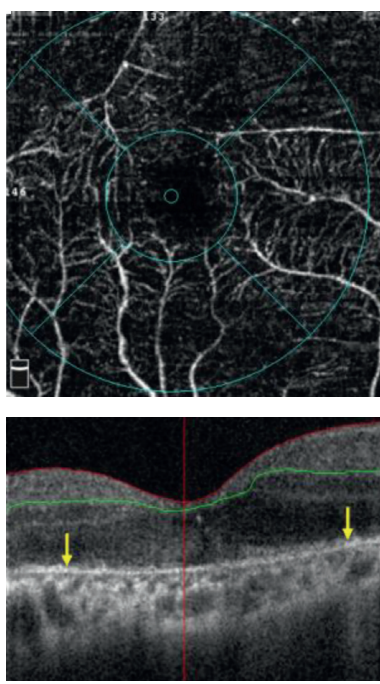

(c)
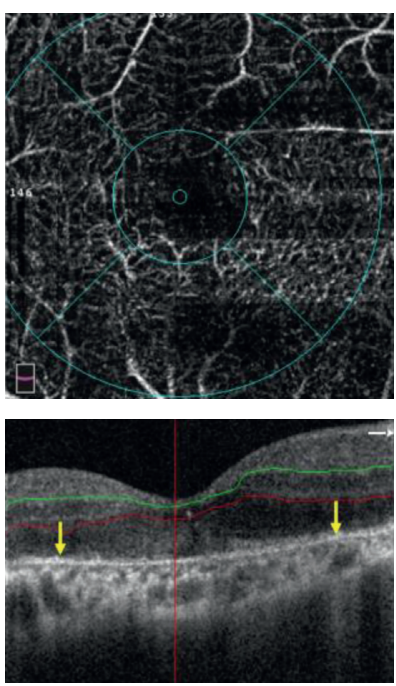

(d)

FIGURE l: Measurement of vessel density in patients with RP. (a) Superior capillary plexus (SCP) of one patient with RP: the ellipsoid zone is intact. (b) Deep capillary plexus (DCP) of one patient with RP: the ellipsoid zone is intact in the macular fovea. Red arrowhead indicates the ellipsoid zone. (c) SCP of one patient with RP: the ellipsoid zone is broken. (d) DCP of one patient with RP: the ellipsoid zone is broken. Yellow arrowhead indicates the ellipsoid zone.

macular intact group, especially in the parafovea region (Table 2). The major difference in VD between the macular intact and macular defect groups was in the DCP, especially in the perifovea region (Table 2). There was no significant difference in SCP between the macular defect and intact groups in either the fovea or parafovea regions $(P=0.296)$. The DCP of the perifovea region showed greater loss of vascular capillary in the macular defect group than in the macular intact group $(P<0.05)$; however, there was no significant difference between the two groups in the fovea and nasal regions $(P=0.296, P=0.127)$. The CMT was found to be lower in patients with RP than in healthy individuals $(P<0.05)$ (Table 2). However, there was no significant difference in the inferior region in healthy individuals and patients with RP $(P=0.147, P=0.453)$. However, the CMT was not significantly different between the macular intact and macular defect groups $(P>0.05)$ (Table 2$)$.

3.3. Correlation between Outer VD and DCP/SCP Ratio. After analysis of the multivariate linear regression, the whole DCP was a dependent variable, and the macular defect and the DCP/SCP ratio in the whole, fovea, and parafovea regions were closely related to the whole DCP $(P<0.05)$ (Table 3). The CRT in the fovea and parafovea regions were not related to whole DCP $(P=0.186, P=0.539)$.

\section{Discussion}

$\mathrm{RP}$ is a broad common inherited disease with photoreceptor degeneration. The present study indicates that the VD decreased in patients with RP, especially in patients with the macular defect. There was a greater loss of vessel plexuses in the DCP when the macular fovea was involved, and the central retinal thickness may not be a more sensitive indicator than the DCP in patients with RP.

A previous study pointed out that retinal perfusion was reduced in patients with RP using the OCTA and FA Doppler ultrasonography $[15,16]$. Several studies found that the reduced VD was mainly in the deep and superficial plexuses. Moreover, some researchers found that decreased retinal perfusion mainly occurred in the parafovea region [6]. Microvascular attenuation was reported in patients with $\mathrm{RP}$, and the reason for microvascular attenuation is caused by the regulatory vasoconstriction and the decreased metabolic demand $[17,18]$. The impaired retinal blood supply might be an early primary event in RP [13]. However, the parts of the retinal vascular supply that were frequently affected were unclear.

In our study, we classified patients with RP into macular intact or defect groups. The results indicated that there was no significant difference in SCP between the two groups, and the major difference was in the DCP. The loss of outer retinal capillaries in the DCP was the main change in the animal of RP [14]. In our study, we classified them into the macular intact or defect group. The results demonstrated that a greater loss of DCP was found in the macular defect group, and the main position for capillary loss in the DCP was in the parafovea region, not the whole and fovea region. The capillary in the DCP was the main nourishment for the outer retinal layers. The decreased retinal perfusion could cause photoreceptor degeneration [13]. A new hypothesis is that outer retinal degeneration could cause vascular remodeling and attenuation [11]. Thus, a greater loss of capillaries in DCP was observed in the macular defect group. Furthermore, why are capillaries in the DCP vulnerable to loss in patients with RP? A previous study demonstrated that more oxygen diffusion 
TABle 1: Demographic information of the study.

\begin{tabular}{|c|c|c|c|c|c|c|}
\hline & \multirow{2}{*}{ Control } & \multicolumn{2}{|c|}{$\mathrm{RP}$} & \multirow{2}{*}{$\begin{array}{c}\text { Control vs. intact } \\
P \text { value }\end{array}$} & \multirow{2}{*}{$\begin{array}{c}\text { Control vs. defect } \\
P \text { value }\end{array}$} & \multirow{2}{*}{$\begin{array}{c}\text { Defect vs. intact } \\
P \text { value }\end{array}$} \\
\hline & & Macular defect & Macular intact & & & \\
\hline Number & 25 & 8 & 9 & & & \\
\hline \multicolumn{7}{|l|}{ Sex (eye/patients) } \\
\hline Male & $10(10)$ & $8(4)$ & $9(5)$ & \multirow{2}{*}{\multicolumn{3}{|c|}{$0.674^{\mathrm{a}}$}} \\
\hline Female & $15(15)$ & $7(4)$ & $6(4)$ & & & \\
\hline Eyes & & 15 & 15 & & & \\
\hline Age, $y$ & $51.0 \pm 12.0$ & $51.3 \pm 12.4$ & $49.0 \pm 10.9$ & 0.736 & 0.959 & 0.744 \\
\hline Range & $(28-66)$ & $(32-60)$ & $(37-57)$ & & & \\
\hline VA (log MAR) & $\begin{array}{c}0 \\
\text { Snellen } 20 / 20 \\
\end{array}$ & $\begin{array}{c}1.5(1.2-0.5) \\
\text { Snellen } 20 / 1000\end{array}$ & $\begin{array}{c}0.3(0.32-0) \\
\text { Snellen } 20 / 40\end{array}$ & $P=0.112$ & $P<0.001$ & 0.014 \\
\hline
\end{tabular}

VA means vision acuity, $P<0.05$ was considered as significant, ANONA for age, a for Chi-square test, and the VA analysis in different groups were compared by Kruskal-Wallis $\mathrm{H}$ test.

TABLE 2: Vessel density and macular retinal thickness in retinitis pigmentosa patients.

\begin{tabular}{|c|c|c|c|c|c|c|c|c|}
\hline & Control & $\mathrm{RP}$ & $P^{\mathrm{a}}$ value & $\begin{array}{c}\text { RP with } \\
\text { macular defect }\end{array}$ & $\begin{array}{c}\mathrm{RP} \text { with } \\
\text { macular intact }\end{array}$ & $\begin{array}{c}\text { Control vs. } \\
\text { intact } \\
P^{\mathrm{b}} \text { value } \\
\end{array}$ & $\begin{array}{c}\text { Control vs. } \\
\text { defect } \\
P^{\mathrm{b}} \text { value }\end{array}$ & $\begin{array}{c}\text { Defect vs. } \\
\text { intact } \\
P^{\mathrm{b}} \text { value }\end{array}$ \\
\hline \multicolumn{9}{|l|}{ SCP } \\
\hline Whole & $53.51 \pm 1.92$ & $32.98 \pm 3.46$ & $<0.001$ & $31.87 \pm 3.16$ & $34.32 \pm 3.66$ & $<0.001$ & $<0.001$ & 0.262 \\
\hline Fovea & $29.28 \pm 2.73$ & $12.19 \pm 8.22$ & $<0.001$ & $14.38 \pm 5.84$ & $9.56 \pm 10.51$ & $<0.001$ & $<0.001$ & 0.359 \\
\hline Parafovea & $55.92 \pm 2.44$ & $35.60 \pm 4.30$ & $<0.001$ & $34.04 \pm 3.98$ & $37.16 \pm 4.42$ & $<0.001$ & $<0.001$ & 0.275 \\
\hline Tempo & $54.53 \pm 1.76$ & $36.34 \pm 6.89$ & $<0.001$ & $36.52 \pm 6.36$ & $36.16 \pm 8.14$ & $<0.001$ & $<0.001$ & 0.094 \\
\hline Superior & $56.85 \pm 2.22$ & $35.82 \pm 6.40$ & $<0.001$ & $34.04 \pm 7.81$ & $37.60 \pm 4.82$ & $<0.001$ & $<0.001$ & 0.411 \\
\hline Nasal & $55.18 \pm 2.28$ & $36.52 \pm 3.14$ & $<0.001$ & $36.02 \pm 3.60$ & $37.12 \pm 2.76$ & $<0.001$ & $<0.001$ & 0.589 \\
\hline Inferior & $56.50 \pm 2.39$ & $33.81 \pm 9.23$ & $<0.001$ & $30.50 \pm 6.72$ & $37.78 \pm 10.97$ & $<0.001$ & $<0.001$ & 0.24 \\
\hline \multicolumn{9}{|l|}{ DCP } \\
\hline Whole & $57.29 \pm 6.63$ & $37.20 \pm 8.64$ & $<0.001$ & $36.55 \pm 1.94$ & $37.98 \pm 13.44$ & $<0.001$ & $<0.001$ & 0.801 \\
\hline Fovea & $28.71 \pm 5.82$ & $23.64 \pm 7.91$ & 0.03 & $21.26 \pm 9.66$ & $26.50 \pm 4.56$ & 0.426 & 0.015 & 0.296 \\
\hline Parafovea & $61.19 \pm 2.18$ & $42.31 \pm 4.29$ & $<0.001$ & $38.96 \pm 1.58$ & $45.66 \pm 3.29$ & $<0.001$ & $<0.001$ & 0.003 \\
\hline Tempo & $60.20 \pm 1.73$ & $41.43 \pm 4.78$ & $<0.001$ & $38.34 \pm 4.52$ & $44.52 \pm 2.70$ & $<0.001$ & $<0.001$ & 0.03 \\
\hline Superior & $44.04 \pm 5.49$ & $62.34 \pm 2.07$ & $<0.001$ & $40.12 \pm 4.69$ & $47.96 \pm 2.72$ & $<0.001$ & $<0.001$ & 0.012 \\
\hline Nasal & $61.22 \pm 2.19$ & $41.16 \pm 5.72$ & $<0.001$ & $38.73 \pm 5.28$ & $44.08 \pm 8.23$ & $<0.001$ & $<0.001$ & 0.127 \\
\hline Inferior & $61.52 \pm 2.26$ & $40.93 \pm 6.56$ & $<0.001$ & $36.55 \pm 4.67$ & $46.18 \pm 4.11$ & $<0.001$ & $<0.001$ & 0.006 \\
\hline \multicolumn{9}{|l|}{ CRT } \\
\hline Fovea & $239.93 \pm 9.59$ & $196.36 \pm 32.11$ & $<0.001$ & $180.50 \pm 35.25$ & $215.40 \pm 13.90$ & $<0.001$ & $<0.001$ & 0.068 \\
\hline Parafovea & $307.31 \pm 9.29$ & $247.70 \pm 29.56$ & $<0.001$ & $236.40 \pm 23.89$ & $259.00 \pm 32.80$ & $<0.001$ & $<0.001$ & 0.248 \\
\hline Temporal & $296.59 \pm 13.02$ & $233.90 \pm 30.68$ & $<0.001$ & $217.20 \pm 21.74$ & $250.60 \pm 30.78$ & $<0.001$ & $<0.001$ & 0.083 \\
\hline Superior & $312.00 \pm 8.43$ & $246.40 \pm 34.68$ & $<0.001$ & $227.20 \pm 23.76$ & $265.60 \pm 34.92$ & $<0.001$ & $<0.001$ & 0.077 \\
\hline Nasal & $310.07 \pm 10.30$ & $261.64 \pm 33.36$ & $<0.001$ & $263.50 \pm 36.51$ & $259.40 \pm 33.23$ & $<0.001$ & $<0.001$ & 0.851 \\
\hline Inferior & $287.80 \pm 76.61$ & $248.55 \pm 33.40$ & 0.124 & $238.83 \pm 28.05$ & $260.20 \pm 38.64$ & 0.147 & 0.453 & 0.315 \\
\hline
\end{tabular}

SCP means superficial capillary plexuses, DCP means deep capillary plexuses, CRT means central retinal thickness, a for independent $t$-test, and $\mathrm{b}$ for LSD.

TABLE 3: Univariable multilevel linear regression analysis for whole DCP.

\begin{tabular}{lccc}
\hline Variable & $\begin{array}{c}\beta \\
\text { coefficient }\end{array}$ & $95 \%$ CI & $P$ value \\
\hline Fovea CRT & -0.146 & -0.181 & 0.186 \\
Parafovea CRT & 0.066 & -0.054 & 0.101 \\
Whole DCP/SCP ratio & 0.784 & $44.826-57.887$ & $<0.001$ \\
Fovea DCP/SCP ratio & 0.164 & $0.019-0.068$ & $<0.001$ \\
Parafovea DCP/SCP & -0.243 & -48.862 to -17.057 & $<0.001$ \\
ratio & 0.909 & $7.55-10.58$ & $<0.001$ \\
Macular $^{\mathrm{a}}$ &
\end{tabular}

CI means confidence interval, CRT means central retinal thickness, $P$ value $<0.05$ was considered significant, DCP means deep capillary plexuses, and SCP means superficial capillary plexuses, a macular intact was considered as the reference term. from the choroid into the superficial retina was detected when the outer retinal thickness decreased $[19,20]$. The capillaries in the SCP were far from the choroid, but when the photoreceptors degenerated, more oxygen was transferred into the DCP, which may cause the dysfunction of the outer retina, and the photoreceptors were prone to degeneration [13]. Moreover, we found that the DCP/SCP ratio was closely related to the whole DCPVD. It stated that the $\mathrm{DCP} / \mathrm{SCP}$ ratio may be an early indicator to reflect the vascular condition in patients with RP. However, there were some limitations in the study, including small samples in each group and without the follow up to detect the changes of vessel density with progression of disease. The magnification of OCT may be affected by the axial length [21, 22]; we have not adjusted the scans to account for the magnification of images. 


\section{Conclusions}

The OCTA could reflect the microvascular changes in either the superficial or deep plexuses in patients with RP. A greater loss of capillaries in the DCP was easily detected when the macular region was involved in patients with RP. The $\mathrm{DCP} / \mathrm{SCP}$ ratio may be an important indicator to reflect the severity of RP.

\section{Data Availability}

The underlying data supporting the results of the study can be obtained upon request to the first author (moses.1988@ 163.com).

\section{Ethical Approval}

This study was approved by the Ethics Committee of Zhejiang Hospital and adhered to the tenets of the Declaration of Helsinki. All the subjects were informed regarding the content of the study before enrollment.

\section{Conflicts of Interest}

The authors declare that there are no conflicts of interest regarding the publication of this article.

\section{Authors' Contributions}

Tingjun Xu designed the study. Jixian Lou and Jiehui Xu collected the data. Jiquan Wen analyzed the data. Mingsheng Hong prepared the manuscript. Mingsheng Hong revised the manuscript.

\section{References}

[1] M. Chizzolini, A. Galan, E. Milan, A. Sebastiani, C. Costagliola, and F. Parmeggiani, "Epidemiologic practice in retinitis pigmentosa: from phenotyping to biobanking," Current Genomics, vol. 12, no. 4, pp. 260-266, 2011.

[2] D. T. Hartong, E. L. Berson, and T. P. Dryja, "Retinitis pigmentosa," Lancet, vol. 18, no. 368, pp. 1795-1809, 2006.

[3] E. L. Berson, M. A. Sandberg, B. Rosner, D. G. Birch, and A. H. Hanson, "Natural course of retinitis pigmentosa over a three-year interval," American Journal of Ophthalmology, vol. 15 , no. 99, pp. 240-251, 1985.

[4] D. Athanasiou, M. Aguila, J. Bellingham et al., "The molecular and cellular basis of rhodopsin retinitis pigmentosa reveals potential strategies for therapy," Progress in Retinal and Eye Research, vol. 62, pp. 1-23, 2018.

[5] K. Konieczka, A. J. Flammer, M. Todorova, P. Meyer, and J. Flammer, "Retinitis pigmentosa and ocular blood flow," The EPMA Journal, vol. 3, no. 1, Article ID 17, 2012.

[6] A. M. Hagag, J. Wang, K. Lu et al., "Projection-resolved optical coherence tomographic angiography of retinal plexuses in retinitis pigmentosa," American Journal of Ophthalmology, vol. 204, pp. 70-79, 2019.

[7] H. R. AttaAllah, A. A. M. Mohamed, and M. A. Hamid, "Quantification of macular microvascular changes in retinitis pigmentosa using optical coherence tomography angiography," Clinical Ophthalmology, vol. 14, no. 14, pp. 1705-1713, 2020.
[8] Y. Jia, O. Tan, J. Tokayer et al., "Split-spectrum amplitude-decorrelation angiography with optical coherence tomography," Optics Express, vol. 20, no. 4, pp. 4710-4725, 2012.

[9] J. Provis, "Development of the primate retinal vasculature," Progress in Retinal and Eye Research, vol. 20, no. 6, pp. 799-821, 2001.

[10] V. A. Barathi, E. Lin, C. Ho et al., "Longitudinal structural and microvascular observation in RCS rat eyes using optical coherence tomography angiography," Investigative Opthalmology \& Visual Science, vol. 61, no. 6, Article ID 54, 2020.

[11] R. L. Pfeiffe, R. E. Marc, and B. W. Jones, "Persistent remodeling and neurodegeneration in late-stage retinal degeneration," Progress in Retinal and Eye Research, vol. 74, Article ID 100771, 2020.

[12] D. Matsunaga, J. Yi, C. A. Puliafito, and A. H. Kashani, "OCT angiography in healthy human subjects," Ophthalmic Surgery, Lasers and Imaging Retina, vol. 45, no. 6, pp. 510-515, 2014.

[13] A. M. Hagag, A. D. Pechauer, L. Liu et al., "OCT angiography changes in the 3 parafoveal retinal plexuses in response to hyperoxia," Ophthalmology Retina, vol. 2, no. 4, pp. 329-336, 2018.

[14] Q. S. You, J. C. H. Chan, A. L. K. Ng et al., "Macular vessel density measured with optical coherence tomography angiography and its associations in a large population-based study," Investigative Opthalmology \& Visual Science, vol. 60, no. 14, pp. 4830-4837, 2019.

[15] R. F. Spaide, J. M. Klancnik, and M. J. Cooney, "Retinal vascular layers imaged by fluorescein angiography and optical coherence tomography angiography," JAMA Ophthalmology, vol. 133, no. 1, pp. 45-50, 2015.

[16] Y. Zhang, J. M. Harrison, O. S. E. Nateras, S. Chalfin, and T. Q. Duong, "Decreased retinal-choroidal blood flow in retinitis pigmentosa as measured by MRI," Documenta Ophthalmologica, vol. 126, no. 3, pp. 187-197, 2013.

[17] A. Finzi, M. Cellini, E. Strobbe, and E. C. Campos, "ET-1 plasma levels, choroidal thickness and multifocal electroretinogram in retinitis pigmentosa," Life Sciences, vol. 118, no. 2, pp. 386-390, 2014.

[18] M. Cellini, E. Strobbe, C. Gizzi, and E. C. Campos, "ET-1 plasma levels and ocular blood flow in retinitis pigmentosaThis article is one of a selection of papers published in the two-part special issue entitled 20 Years of Endothelin Research," Canadian Journal of Physiology and Pharmacology, vol. 88, no. 6, pp. 630-635, 2010.

[19] J. S. Penn, S. Li, and M. I. Naash, “Ambient hypoxia reverses retinal vascular attenuation in a transgenic mouse model of autosomal dominant retinitis pigmentosa," Investigative Ophthalmology \& Visual Science, vol. 41, no. 12, pp. 4007-4013, 2000.

[20] L. Padnick-Silver, J. J. K. Derwent, E. Giuliano, K. Narfstrom, and R. A. Linsenmeier, "Retinal oxygenation and oxygen metabolism in abyssinian cats with a hereditary retinal degeneration," Investigative Opthalmology \& Visual Science, vol. 47, no. 8, pp. 3683-3689, 2006.

[21] D. M. Sampson, P. Gong, D. An et al., "Axial length variation impacts on superficial retinal vessel density and foveal avascular zone area measurements using optical coherence tomography angiography," Investigative Opthalmology \& Visual Science, vol. 58, no. 7, pp. 3065-3072, 2017.

[22] J. Chua and L. Schmetterer, "Letter to the Editor on Macular OCT-angiography parameters to predict the clinical stage of nonproliferative diabetic retinopathy: an exploratory analysis," Eye, vol. 34, no. 12, pp. 2341-2342, 2020. 